\title{
We Yolande Govindama (1951-2020)
}

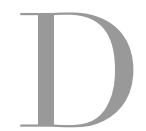

écédée tragiquement sur une route de Normandie, inhumée à Saint-Pierre le 8 novembre 2020, Yolande Govindama était auteure, directrice de laboratoire, titulaire de la chaire de psychologie clinique à la faculté de Rouen. Élève d'Hélène Stork, anthropologue réputée Yolande avait réalisé sa thèse, sous la direction de celle-ci, sur l'enfance en pays tamoul.

J'ai rencontré Yolande en 1988, depuis nous sommes toujours restés en lien. Cette année 1988, le médecin psychiatre Jean-François Reverzy qui avait décidé de s’installer sur l'île de la Réunion projeta d'y faire un immense colloque “ L'espoir transculturel » dédié à la mémoire de Dulcie September militante anti-apartheid sud africaine assassinée le 29 mars 1988 dans des conditions encore couvertes par l'obscurité à Paris ${ }^{[1]}$. Si les noms composant les comités d'organisation et scientifique remplissaient une pleine page de bottin, il est vrai que l'efficace de l'organisation tenait vraiment entre les mains de Yolande toujours très affairée et attentive comme elle n'a cessé de l'être.

Le ton de mon hommage est donné : mon compagnonnage intellectuel avec Yolande n'a pas d'abord été universitaire, il a plutôt été militant et il l'est resté. En effet, peu d'années après ce moment étonnant à la Réunion, j’ai participé à “ La route de l'esclave " - un projet de l'Unesco- avec des amis antillais que je connaissais depuis 1980, et des amis sénégalais. J'ai, par la suite, à la fin des années 1990, monté avec Jean-Marc Masseaut, Hughes Liborel-Pochot et Dany Bébel-Gisler une revue qui fait toujours parler d'elle, "Les cahiers de la mémoire ", revue que nous portâmes à sur les fonds baptismaux à Dakar et qui pris vite siège à Nantes.

Quel rapport tout ceci avec Yolande? C'est que Yolande assez régulièrement et parmi ses multiples activités, animait souvent à l'Unesco des tables rondes sur l'abolition de l'esclavage au moment où on célébrait la deuxième abolition, et, généreusement, elle m'invita à trois reprises à m’y exprimer. Ces temps de célébration nous ont vraiment beaucoup rapprochés et permirent que se nouèrent par la suite des échanges universitaires. Je me souviens bien d'elle dans des colloques, elle était toujours passionnée ; elle ne cédait pas sur l'essentiel mais elle était courtoise avec les gens qui s'exprimaient alors qu'elle avait des positions tranchées et courageuses très différentes des fadaises et des opinions courantes.

[1] Elle fut abattue sur le palier des bureaux de l'ANC au 4 étage du 28 rue des Petites Écuries de cinq balles tirées à bout portant d'un calibre 22 équipé d'un silencieux d'une façon professionnelle. 
J'insiste aussi sur mon bonheur de directeur de publication de la revue Psychologie Clinique, car lisant un article de Yolande avant de le soumettre à expertise, non seulement je goutais une prose et une présentation impeccablement nettes et lisait en chacun de ces articles (dont aucun ne fut refusé) l'expression de cet altruisme, de ce besoin de transmettre qu'elle mettait en ouvre en demandant à des thésards et thésardes de co-signer des articles dans lesquels chacun des signataires s'engageaient à part entière.

J'ajoute que j'ai eu le bonheur d'être invité par Yolande à des soutenances de thèses à Rouen. Qu'elle ait été directrice de la thèse ou simple membre du jury, Yolande, n'était pas toujours gentille, non, mais elle était extrêmement exigeante et toujours dans une bonté accueillante. Comme très peu mais vraiment très peu d'enseignants dès que Yolande avait décidé de suivre et de diriger un travail, un mémoire ou $a$ fortiori une thèse, son étudiant était déjà admis dans une communauté de chercheurs de sorte que la relation directeur de thèse - thésard n'était pas strictement une relation hiérarchique du type Maitre- élève mais une relation plutôt initiatique au sein de laquelle chacun pouvait trouver son style et sa voie propre.

Elle s'intéressait donc vraiment à la transmission dans un mode non conservatoire et transdisciplinaire résolu et assumé. La méthode anthropologique elle la connaissait par le cinéma et elle s’inscrivait ans l'orbe magistral qu'avait dessiné Jean Rouch. Ses films sont absolument extraordinaires de rythme et de précision, elle ne filme pas comme Rouch, mais elle ne filme pas sans Rouch, faisant preuve d'un grand sens du cadrage et sachant prendre son temps pour que le surprise advienne. Nous pouvions alors, nous spectateurs, lire dans les moments ritualisés de passage ce que représente pour certains sujets en décalage culturel, en transition, le poids d'éprouver la pression du collectif sur leur singularité. Par quoi son art se rapproche des trop confidentiels films de Jaak le Roy portant sur les églises de guérison à Kinshasa.

On pourrait, ici confisquer la présence de Yolande dans un immense catalogue de ses multiples dons, de ses multiples curiosités, de la fécondité de ses recherches et de l'impressionnante collection de ses terrains de recherches. Cela nous priverait de saisir que Yolande avait un style fait de rigueur, d'inquiétude et elle fait partie des gens qui étaient très vigilant sur les effets subjectifs et collectifs des logiques de ségrégations. Elle rejoignait ceux des ethnopsychiatres qui ne sont pas partisans valoriser la coutume au détriment de la raison républicaine. Et son érudition anthropologique était vaste. Sa méthode était tout aussi rigoureuse qu'elle porte intérêt aux relations mères-enfants en Afrique ou dans son île natale qu'elle décrive comme peu purent et surent le faire les rituels Tamouls. Le choix de refuser toute mascarade identitaire tout en explorant et dénonçant les violences coloniales n'était pas sans conséquence ; la clarté intellectuelle et morale d'un tel choix manque de nos jours. J'ajoute qu'elle cultivait une saveur de l'amitié et de la provocation de bon ton entre camarades de longue date; elle me taquinait sur mon lacanisme et pourtant elle a 
pris de Lacan ce qui lui interdisait d'être de trop dans le culturalisme, et trouvait un ressort universaliste dans sa lecture au tripode lacanien RSI.

Yolande essayait de voir comment un trait culturel pouvait être utilisé de façon dommageable, pathologique parfois, elle retrouvait là une grande thèse de Devereux. La deuxième chose qu'elle avait retenue de l'enseignement de Devereux ouvre sur la question fascinante et mélancolique qui est celle de a haine de l'identité et du renoncement à l'identité et cela la préoccupait beaucoup car qui se dépersonnalise pour rentrer dans le commun ne peut plus être un héritier. Yolande était concernée au plus haut point par ce que nous appelions " une mission ontologique de transmission ». Acteur politique et scientifique qui respectait les traditions, les coutumes, les cultures sans les fétichiser, elle donnait à chaque personne qu'elle avait en cure ou qu'elle rencontrait dans ses recherches, la possibilité de ne pas être honteux de ses héritages les plus violents et les plus cachés qu’ils puissent être. Elle permettait à chacun la possibilité fière et digne d'être un agent de transmission et de métamorphose du passé.

C'est en tout cas l'impression la plus forte qu'elle me fait. Aujourd'hui, je perds une amie, mais le fait de pouvoir écrire ces quelques lignes me rassure; je sais que sa pensée et ses engagements continueront leurs chemins et porteront leurs fruits

\section{Olivier Douville}

\section{Recherches menées :}

- à la Réunion (thèse sur la fonction des rites de passage et thérapeutiques en santé mentale ; contrat INSERM-DOM : troubles du sommeil de l'enfant; Révision de la classification internationale des maladies mentales avec l'OMS, etc.);

- avec l'université d'Harvard (codirection de thèse : maternage et culture);

- avec l'université de Stanford (contrat de recherche international : émotion et culture);

- avec l'université de Mostaganem (Algérie) convention avec l'université de Rouen (Dépression et culture et dispositifs de soins);

- avec l'université d'Oran (Algérie), convention avec l'université de Rouen (autisme et culture, soins);

- direction des thèses depuis 2003 ; des docteurs en psychologie sont déjà nommés maîtres de conférences en France et dans leur psys d'origine.10 thèses en cours dont 3 sur la Réunion et une en codirection avec l'université de la Réunion (thèmes : violence intrafamiliale et histoire ; mobilité et abandon d'enfant; la langue créole et le culte des ancêtres dans une fonction d'arrimage à une fonction paternelle).

- Publications et colloques (France, Suisse, Portugal, Brésil, Canada, USA, Burkina Faso, Sénégal, Martinique, Inde, La Réunion, Israël, Algérie). 


\section{Enseignement :}

- Université de Ouagadougou (Burkina Faso) dès 2005-Mise en place du diplôme de psychiatre avec le soutien du ministère de la coopération française.

- Université Aberta de Lisbonne (Portugal)). Enseigne depuis 2003 dans le cadre ERAMUS (mobilité des enseignants).

- Université de Mostaganem (Algérie)-mise en place du LMD en psychologie.

\section{Références :}

2017 - Maltraitance et désir d'infanticide (coll. sous la direction de Y. G.), Paris, Khartala. 2011 - Temps et rite de passage (coll. sous la direction de Y. G.), Paris, Khartala.

2006 - Le monde hindou à la réunion. Une approche anthropologique et psychanaytique, Paris, Khartal.a 